\title{
Assessing severe acute respiratory coronavirus virus 2 (SARS-CoV-2) preparedness in US community hospitals: A forgotten entity
}

\author{
Sonali D. Advani MBBS, MPH ${ }^{1,2}$ (1) Esther Baker MSN, RN, CIC ${ }^{1}$, Andrea Cromer BSN, RN, MT, MPH, CIC, CPH ${ }^{1}$, \\ Brittain Wood BSN, RN, CRCST, CIC ${ }^{1}$, Kathryn L. Crawford BSBA-HCM, RN, CIC ${ }^{1}$, Linda Crane BSMT (ASCP) SM, CIC ${ }^{1}$, \\ Linda Adcock RN, BSN, $\mathrm{CIC}^{1}$, Linda Roach BSMT, CIC, CCHM ${ }^{1}$, Polly Padgette RN, BSN, CIC, FAPIC ${ }^{1}$, \\ Deverick J. Anderson MD, MPH ${ }^{1,2}$, Daniel J. Sexton MD ${ }^{1,2}$, for the CDC Prevention Epicenter Program \\ ${ }^{1}$ Duke Center for Antimicrobial Stewardship and Infection Prevention, Durham, North Carolina and ${ }^{2}$ Duke University School of Medicine, Department of Internal \\ Medicine, Durham, North Carolina
}

\begin{abstract}
We performed a cross-sectional survey of infection preventionists in 60 US community hospitals between April 22 and May 8, 2020. Several differences in hospital preparedness for SARS-CoV-2 emerged with respect to personal protective equipment conservation strategies, protocols related to testing, universal masking, and restarting elective procedures.
\end{abstract}

(Received 13 July 2020; accepted 27 September 2020; electronically published 7 October 2020)

Novel coronavirus (SARS-CoV-2) has been associated with the largest recorded coronavirus outbreak to date. In the United States, there have been $>2.2$ million cases with $>119,000$ deaths (as of June 21, 2020). ${ }^{1}$ This pandemic has placed a tremendous strain on the US healthcare system leading to personal protective equipment (PPE) and resource shortages. ${ }^{2}$ Most hospitals have implemented contingency and crisis capacity strategies to optimize the use of resources. ${ }^{3}$

Although public health agencies like the Centers for Disease Control and Prevention (CDC) have provided interim guidance on infection prevention and control in US hospitals, ${ }^{4}$ the current state of community hospital preparedness is unknown. Assessing preparedness of community hospitals is crucial to risk assessments and outbreak control activities in these settings. Hence, we conducted a cross-sectional survey of SARS-CoV-2 preparedness among community hospitals in southeastern United States.

\section{Methods}

\section{Survey design and setting}

We performed a cross-sectional survey of 60 community hospitals within the Duke Infection Control Outreach Network (DICON). DICON provides infection control services to 60 community hospitals and surgery centers in 6 states (North Carolina, South

Author for correspondence: Sonali D. Advani, E-mail: sonali.advani@duke.edu PREVIOUS PRESENTATION. These data were presented in part as "SARS-CoV-2 Preparedness among Community Hospitals in Southeastern United States" (abstract 902199) at IDWeek 2020 on October 21-22, 2020, virtually, in Boston, Massachusetts.

Cite this article: Advani SD, et al. (2021). Assessing severe acute respiratory coronavirus virus 2 (SARS-CoV-2) preparedness in US community hospitals: A forgotten entity. Infection Control \& Hospital Epidemiology, 42: 600-603, https:// doi.org/10.1017/ice.2020.1238
Carolina, Virginia, Florida, Georgia, and West Virginia). ${ }^{5}$ These hospitals range in size from 30 to 685 beds, with a median size of 162 beds. Also, 77\% of these hospitals have maternity and pediatric wards. This study was deemed exempt from institutional review board review by the Duke University Health System (no. Pro00105818).

\section{Survey instrument and distribution}

The survey (provided in the Supplementary Data online) was conducted between April 22 and May 5, 2020, using Qualtrics (Qualtrics, Provo, UT); it was distributed electronically to infection preventionists at community hospitals. Participation was voluntary, anonymous, and without compensation. The survey included 13 questions related to PPE availability, crisis capacity strategies to extend and reuse PPE, policies related to restarting surgeries, testing prior to elective surgery and prior to transfer to extended care facilities, universal masking, and daily screening of hospital staff. Extended use was defined as using the same single-use PPE for encounters with multiple patients without removing it between encounters. Reuse was defined as using the same PPE for multiple encounters but doffing it after each encounter and donning it prior to the next encounter. Survey responses were analyzed using descriptive statistics.

\section{Results}

Of 60 hospitals, 50 (83\%) responded to our survey. These hospitals reported varying degrees of PPE shortages (Fig. 1). Overall, 20 hospitals (40\%) reported "no supply" or "few days supply" of powered air-purifying respirators (PAPRs), environmental disinfectant, and gowns. Almost $30 \%$ of facilities reported an 


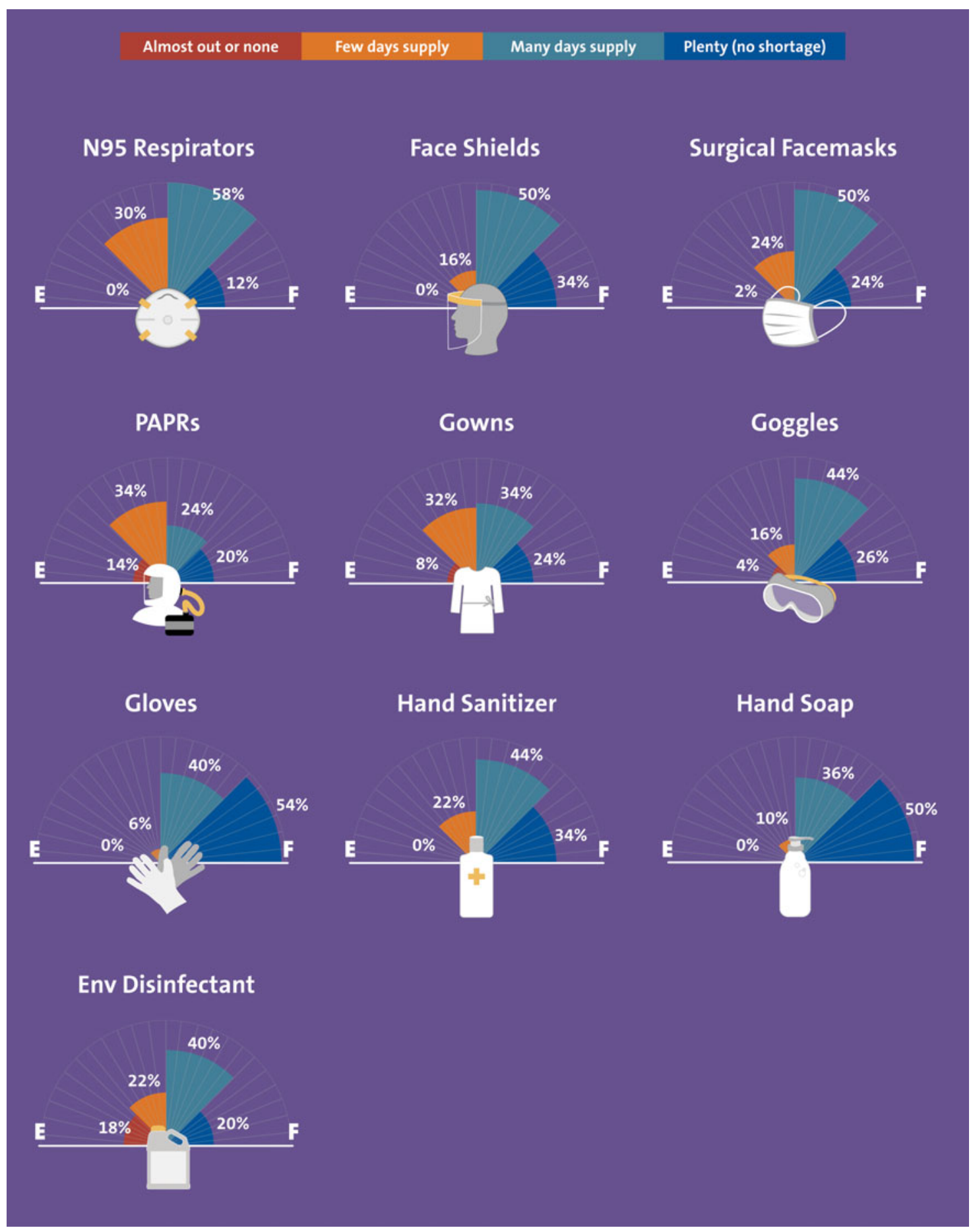

Fig. 1. Supply of resources in 50 community hospitals in the southeastern United States.

insufficient supply of face masks and N95 respirators, and 16\% reported an insufficient supply of face shields. More than $80 \%$ of community hospitals were implementing strategies to reuse N95 respirators, face shields, and goggles. Only 6 hospitals (12\%) were reusing gowns at the time of this survey. Similarly, at least $80 \%$ of hospitals were extending the use of $\mathrm{N} 95$ respirators, face shields, and surgical masks (Table 1). Furthermore, $36 \mathrm{com}$ munity hospitals $(72 \%)$ reported reprocessing N95 respirators, mostly using hydrogen peroxide plasma (29.8\%), ultraviolet radiation $(21 \%)$, and/or hydrogen peroxide vapor (12\%).
Most community hospitals had implemented universal masking policies: 38 (76\%) required masking of patients, visitors and healthcare personnel (HCPs), 7 (14\%) required masking of HCPs and visitors; and $4(8 \%)$ required universal masking of HCPs only. Also, $90 \%$ of hospitals were performing daily employee screening at point of entry. Additionally, 7 (14\%) hospitals had restarted tier 1 elective surgical procedures at the time of this survey; $16(32 \%)$ restarted tier 2 nonurgent surgical procedures; and $43(86 \%)$ were performing only tier 3 emergent surgical procedures. Only 43 facilities (86\%) reported performing 
Table 1. Distribution of Community Hospitals That Extend the Use of Their Personal Protective Equipment (PPE) or Reuse Their PPE

\begin{tabular}{lcc}
\hline Type of PPE & Reuses PPE, No. (\%) & Extends PPE, No. (\%) \\
\hline N95 respirators & $43(86)$ & $44(88)$ \\
\hline Face shields & $46(92)$ & $40(80)$ \\
\hline Surgical facemasks & $32(64)$ & $38(76)$ \\
\hline Gowns & $6(12)$ & $10(20)$ \\
\hline Gloves & $0(0)$ & $2(4)$ \\
\hline Goggles & $41(82)$ & $30(60)$ \\
\hline PAPRs & $38(76)$ & $25(50)$ \\
\hline
\end{tabular}

Note. PAPR, powered air-purifying respirator.

preoperative testing for SARS-CoV-2. Moreover, 17 facilities (34\%) performed 1 SARS-CoV-2 PCR test before discharging an asymptomatic patient to skilled nursing facilities, and 20 facilities (40\%) performed 2 tests prior to discharge to these facilities. The community hospitals in our network reported a wide variety of laboratories used for SARS-COV-2 testing, with most using in-house testing $(n=34,68 \%)$, followed by testing by LabCorp $(\mathrm{n}=21,42 \%)$, Quest Diagnostics $(\mathrm{n}=13,42 \%)$, Department of Health $(\mathrm{n}=13,26 \%)$, and others. Only $4 \%$ of hospitals performed antibody testing for SARS-COV-2 at the time of this survey.

\section{Discussion}

The results of this survey reveal gaps and differences in SARS-COV-2 preparedness among community hospitals in the southeastern United States. A recent survey of hospitals in the Society for Healthcare Epidemiology of America Research Network highlighted similar shortages in academic hospitals and large medical centers, ${ }^{6}$ but our survey is the first report, to our knowledge, focusing on the state of smaller community hospitals during the COVID-19 pandemic.

Almost half of the community hospitals reported shortages in their supplies of PAPRs, environmental disinfectants, and gowns. In addition, $80 \%$ of hospitals reported an adequate supply of N95 respirators, face shields, and googles, likely due to use of crisis capacity strategies to extend, reuse, and reprocess these PPE. Our report is different from a recently reported survey of hospitals in Idaho that reported shortages of face shields. ${ }^{7}$ Our survey highlights that face shields are less prone to shortages due to their simpler design, reuse potential, and durability. ${ }^{8}$

More than half of the community hospitals in our network had employed strategies to extend the use of face masks, N95 respirators, gowns, face shields, and googles. Currently, to our knowledge, no data are available on the safety of extended use PPE or time limits for safely extending the use. Similarly, most hospitals were employing strategies to reuse N95 respirators, PAPRs, face shields, goggles, and masks. Although some data exist on the safety of reprocessed N95 respirators, safety data on reuse of other single use PPE are scarce. ${ }^{9}$ Shortages of disinfectants and sanitizers may lead to the introduction of new agents with a potential decrease in cleaning efficiency, variation in equipment compatibility, an increase in staff dissatisfaction, and occupational safety hazards. ${ }^{9}$

Our survey also demonstrates that most of our community hospitals had implemented policies related to employee screening at the point of hospital entry. Although most hospitals had developed policies related to universal masking, the content of these policies varied widely. There was significant variation in policies related to testing for active infection with SARS-CoV-2 infection, with respect to the laboratory used, testing before surgical procedures, and testing prior to discharge to skilled nursing facilities.

Our study has several limitations. It was a cross-sectional study and relied on self-reported data from infection preventionists. We did not include other healthcare facilities such as nursing homes. However, this survey provided valuable information on differences in outbreak readiness among community hospitals that may help identify factors influencing preparedness.

We found several differences in community hospital preparedness for SARS-CoV-2 with respect to type of conservation strategies used to preserve PPE, protocols related to testing, masking, and restarting elective procedures. We believe that this lack of standardization in approaches was due to differences in state guidelines, the decentralized federal approach to SARS-CoV-2 preparedness, and a lack of confidence in public health guidelines. These differences also highlight the challenges with implementing guidelines related to SARS-CoV-2 in community hospitals because of PPE and personnel shortages, financial constraints, and uncertainty regarding how and when to implement policies such as universal masking, preoperative testing, and predischarge testing. This study also offers a starting point for future assessments of pandemic preparedness among community hospitals in the United States.

Supplementary material. To view supplementary material for this article, please visit https://doi.org/10.1017/ice.2020.1238

Acknowledgments. We thank the community hospitals in our network for participating in this survey.

Financial support. This work was funded by the Centers for Disease Control and Prevention (grant no. 6U54CK000483).

Conflicts of interest. Dr Advani reports grants from the CDC. Dr Anderson reports grants from the AHRQ, the CDC, and the NIAID, as well as personal fees from UpToDate, outside the submitted work. All other authors report no conflicts of interest or disclosures relevant to this article.

\section{References}

1. Cases in the United States. Centers for Disease Control and Prevention website. https://www.cdc.gov/coronavirus/2019-ncov/cases-updates/cases-in-us.html? CDC_AA_refVal=https\%3A\%2F\%2Fwww.cdc.gov\%2Fcoronavirus\%2F2019ncov\%2Fcases-in-us.html. Published June 21, 2020. Accessed June 21, 2020.

2. Bauchner $\mathrm{H}$, Fontanarosa $\mathrm{PB}$, Livingston $\mathrm{EH}$. Conserving supply of personal protective equipment-a call for ideas. JAMA 2020. doi: 10.1001/jama.2020. 4770 .

3. Rowan NJ, Laffey JG. Challenges and solutions for addressing critical shortage of supply chain for personal and protective equipment (PPE) arising from Coronavirus disease (COVID19) pandemic — case study from the Republic of Ireland. Sci Total Environ 2020;725:138532.

4. Interim infection prevention and control recommendations for patients with suspected or confirmed coronavirus disease 2019 (COVID-19) in healthcare settings. Centers for Disease Control and Prevention website. https://www. cdc.gov/coronavirus/2019-ncov/hcp/infection-control-recommendations.html. Published June 19, 2020. Accessed June 20, 2020.

5. Duke Infection Control Outreach Network website. https://dicon.medicine. duke.edu/about. Published 2020. Accessed 2020, April 1.

6. Calderwood MS, Deloney VM, Anderson D, et al. Policies and practices of SHEA research network hospitals during the COVID-19 pandemic. Infect Control Hosp Epidemiol 2020. doi: 10.1017/ice.2020.303. 
7. Kanwar A, Heppler S, Kanwar K, Brown CK. A survey of COVID-19 preparedness among hospitals in Idaho. Infect Control Hosp Epidemiol 2020. doi: $10.1017 /$ ice. 2020.218

8. Advani SD, Smith BA, Lewis SS, Anderson DJ, Sexton DJ. Universal masking in hospitals in the COVID-19 era: is it time to consider shielding? Infect Control Hosp Epidemiol 2020. doi: 10.1017/ice.2020.179.
9. Berardi A, Perinelli DR, Merchant HA, et al. Hand sanitisers amid COVID-19: a critical review of alcohol-based products on the market and formulation approaches to respond to increasing demand. Int $J$ Pharm 2020;584:119431. 